\title{
Heart rate recovery after exercise: relations to heart rate variability and complexity
}

M. Javorka, I. Žila,

T. Balhárek and K. Javorka
Department of Physiology, Jessenius Faculty of Medicine, Comenius University, Martin, Slovak Republic

\section{Correspondence}

M. Javorka

Department of Physiology, JLF UK

Malá Hora 4

036-01 Martin

Slovakia

Fax: +421-43-422-2260

E-mail: mjavorka@jfmed.uniba.sk

Received January 18, 2002

Accepted June 18, 2002

\section{Abstract}

Physical exercise is associated with parasympathetic withdrawal and increased sympathetic activity resulting in heart rate increase. The rate of post-exercise cardiodeceleration is used as an index of cardiac vagal reactivation. Analysis of heart rate variability (HRV) and complexity can provide useful information about autonomic control of the cardiovascular system. The aim of the present study was to ascertain the association between heart rate decrease after exercise and HRV parameters. Heart rate was monitored in 17 healthy male subjects (mean age: 20 years) during the pre-exercise phase ( $25 \mathrm{~min}$ supine, $5 \mathrm{~min}$ standing), during exercise ( $8 \mathrm{~min}$ of the step test with an ascending frequency corresponding to $70 \%$ of individual maximal power output) and during the recovery phase (30 min supine). HRV analysis in the time and frequency domains and evaluation of a newly developed complexity measure - sample entropy - were performed on selected segments of heart rate time series. During recovery, heart rate decreased gradually but did not attain pre-exercise values within $30 \mathrm{~min}$ after exercise. On the other hand, HRV gradually increased, but did not regain rest values during the study period. Heart rate complexity was slightly reduced after exercise and attained rest values after 30min recovery. The rate of cardiodeceleration did not correlate with pre-exercise HRV parameters, but positively correlated with HRV measures and sample entropy obtained from the early phases of recovery. In conclusion, the cardiodeceleration rate is independent of HRV measures during the rest period but it is related to early postexercise recovery HRV measures, confirming a parasympathetic contribution to this phase.

\section{Introduction}

During exercise, an increase of sympathetic activity and a decrease of vagal discharge lead to an increase of heart rate, stroke volume, and myocardial contractility to satisfy energy demands of working muscles. Exercise cardioacceleration results from release

\section{Key words}

- Exercise recovery

- Heart rate variability

- Entropy

- Spectral analysis

- Parasympathetic system 
cise stimulus from the brain (cerebral cortex - central command) which is responsible for the initial rapid drop of heart rate (2). Slower changes in the stimuli to metaboreceptors and baroreceptors accompanying clearance of metabolites and delayed elimination of body heat and catecholamines are thought to be other factors contributing to heart rate recovery after physical activity. Nevertheless, parasympathetic activation is considered to be the main mechanism underlying exponential cardiodeceleration after exercise (1-4).

The rate of decrease in heart beat frequency and the length of time to recovery after moderate-to-heavy exercise are commonly used as indicators of cardiovascular fitness (5). Recently, a delayed decrease in heart rate during the first minute after exercise has been suggested to be a powerful and independent predictor of all-cause mortality $(3,6,7)$.

A modern and perspective approach to the study of physiological control system reactions to physical activity is the assessment of heart rate variability (HRV), mainly before and immediately after the physical load (8). The parameters of HRV in time and frequency domains can provide useful information about the cardiovascular system control and have also been found to be independent predictors of mortality in a number of prospective epidemiological studies (for a review, see Ref. 9). Application of new parameters based on nonlinear dynamics can provide additional information about systems involved in the control of cardiovascular parameters which are undetectable by conventional linear HRV analysis. Sample entropy - the measure of system complexity and unpredictability - is one of them (10).

Because of the parasympathetic contribution to both HRV and heart rate recovery, we hypothesized that the HRV indices (predominantly of parasympathetic activity) before and after exercise will be associated with the rate of cardiodeceleration after acute dynamic physical activity. Therefore, the principal aim of the present study was to ascertain the association between heart rate recovery after exercise, HRV and heart rate complexity.

\section{Material and Methods}

\section{Subjects}

The study was performed on 17 healthy untrained male volunteers (age $20.3 \pm 0.2$ years, body mass index $23.9 \pm 0.5 \mathrm{~kg} / \mathrm{m}^{2}$ ) All subjects were asked to avoid smoking and drinking alcoholic beverages before the experimental procedures and none of them were taking any medication known to affect cardiovascular function.

The protocol was approved by the Faculty Ethics Committee and all participants gave informed consent.

\section{Procedures}

The experimental protocol consisted of two sessions performed on separate days. On the first day individual maximal power output was determined to standardize the exercise level within the study group on the second measurement day.

Maximal power output. Submaximal prediction procedure by the method of Maritz (according to Ref. 1) was used to determine maximal power output (Wmax). All subjects performed the step test - repeated climbing on a bench (height $0.46 \mathrm{~m}$ ) - with four increasing frequencies with each work-rate stage lasting $3 \mathrm{~min}$. Heart rate at the end of each work-rate stage (during the heart rate steady-state achieved in the last minute of a given stage) was plotted against the corresponding power output (product of body weight, gravity constant, step height and frequency of ascents). After extrapolation, Wmax was estimated as a power output associated with predicted maximum heart rate (based on the formula $220\left(\mathrm{~min}^{-1}\right)$ - age). 
Experimental session. On the subsequent day, participants were instructed to lie quietly in the supine position (L-phase). After $25 \mathrm{~min}$ in the supine position the subjects were asked to stand up slowly and to remain in the standing position for $5 \mathrm{~min}$ (S-phase). Next, the step test with a frequency corresponding to $70 \%$ of individual Wmax and duration of $8 \mathrm{~min}$ was performed during the exercise phase (E-phase). Next, subjects rested for $35 \mathrm{~min}$ in the supine position for recovery (R-phase). Subjects breathed spontaneously throughout the experimental procedure with no attempt to control the depth or frequency of the respiratory pattern.

\section{Data acquisition and analysis}

During both sessions (determination of Wmax and experimental session) heart rate, represented by its reciprocal value ( $R R$ interval), was monitored beat-to-beat using a telemetric ECG system (Sima Media, Olomouc, Czech Republic) with a sampling rate of $1000 \mathrm{~Hz}$. Rare premature beats were replaced by linear interpolation of adjacent beats.

Heart rate variability. Subsequent HRV analysis in time and frequency domains on selected segments of the record (with a length of $250 \mathrm{~s}$ ) was performed off-line using a special software (Figure 1). During the Lphase (subject lying supine before exercise) we analyzed HRV in five segments (L1-L5), with the L1 segment starting $5 \mathrm{~min}$ after reclining and the L5 segment ending $10 \mathrm{~s}$ before the subsequent phase. The S-phase was considered as a separate segment but the first $50 \mathrm{~s}$ were ignored to eliminate shortterm heart rate changes upon standing. Recovery (R-phase) was divided into five segments (R1 - 300-550 s, R2 - 600-850 s, R3 900-1150 s, R4 - 1200-1450 s, and R5 1500-1750 s after the cessation of exercise). We omitted the E-phase from the HRV analyses due to frequently occurring artifacts caused by subject movements during the step test. At the onset of recovery (0-300 s after cessation of exercise) HRV was not quantified because of time series nonstationarity.

Selected time domain parameters, i.e., mean duration of RR interval (mean RR interval), standard deviation of RR intervals (SDRR), the square root of the mean squared difference of successive RR intervals (RMSSD), and the proportion of interval differences of successive RR intervals greater than $50 \mathrm{~ms}$ (pNN50), were computed from raw RR intervals.

Spectral analysis was performed on linearly resampled $(2 \mathrm{~Hz})$ time series. Then, the 256-point fast Fourier transformation was repeatedly computed within a selected segment of recording (length of $250 \mathrm{~s}$, corresponding to 500 samples after resampling) with shift of 10 points. The significant trend in each window analyzed was removed by subtracting from the time series the bestfitting regression line and Hanning window was applied to avoid spectral leakage. Subsequently, the mean spectrum of the analyzed segment was computed and spectral power in low $(0.05-0.15 \mathrm{~Hz}, \mathrm{LF})$ and high frequency bands $(0.15-1.00 \mathrm{~Hz}, \mathrm{HF})$ was obtained by integration. Following the rec-

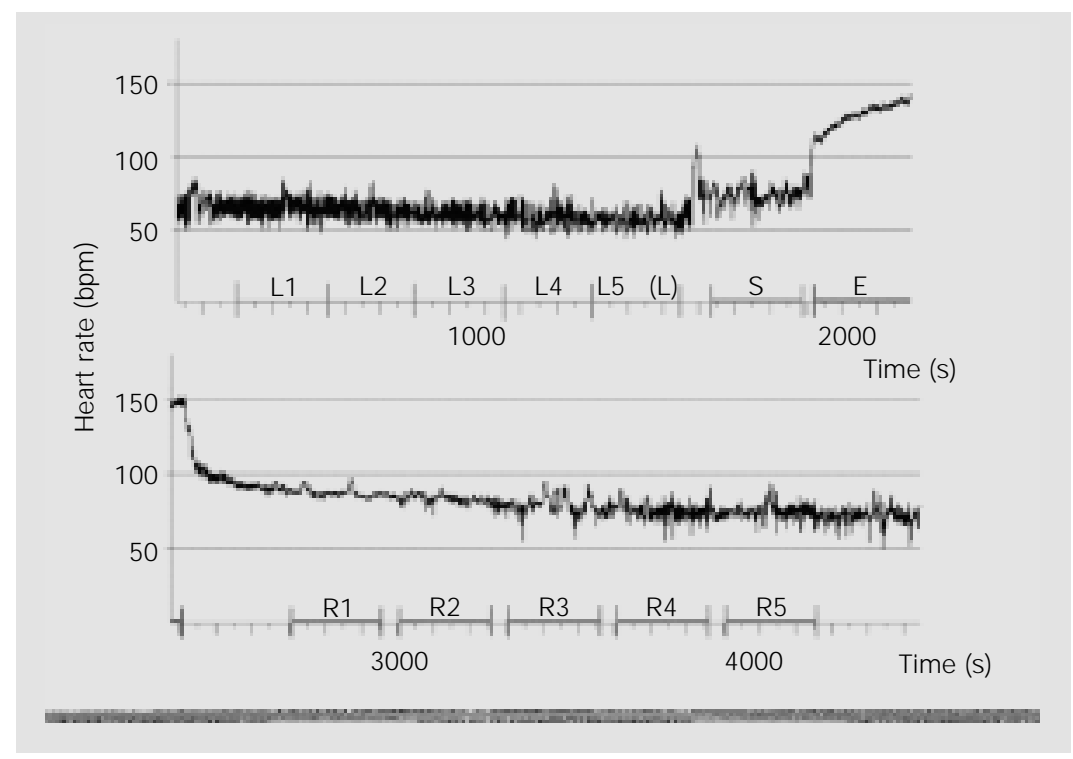

Figure 1. Original record of heart rate changes during the experiment. The segments which we analyzed are indicated (for further description, see Material and Methods). 
ommendations of the Task Force of the European Society of Cardiology and the North American Society of Pacing and Electrophysiology (9), we omitted analysis of the very low frequency band (under $0.05 \mathrm{~Hz}, \mathrm{VLF}$ ) due to the controversial physiological explanation of heart rate fluctuations in this band and the short length of the analyzed window.

A recently developed parameter quantifying complexity and regularity of heart rate time series called sample entropy, whose algorithm was published elsewhere (10), was computed on 250 points of analyzed segments after $1 \mathrm{~Hz}$ resampling. Parameter $m$ was fixed to 2 and tolerance level $r$ was 0.2 times the standard deviation of the analyzed window to allow measurements and comparisons of data sets with different overall variabilities (11).

Heart rate decrease during the recovery phase was quantified as percent heart rate decrease from the peak exercise heart rate level $(100 \%)$ during the 1 st min of recovery $(\% \mathrm{D} 1)$.

\section{Statistical analysis}

Logarithmic transformation was performed on LF and HF spectral powers because they did not show a normal distribution. Repeated measures ANOVA with contrasts was used to determine changes in the parameters assessed during the experimental session. Pearson correlations were computed on selected pairs of parameters. All inferential and correlation statistics were considered significant at $\mathrm{P}<0.05$ and values are reported as means $\pm \mathrm{SEM}$.

\section{Results}

\section{Submaximal determination of Wmax}

All participants completed submaximal step test determination of Wmax according to the Maritz method (1) to standardize testing exercise level at $70 \%$ Wmax. The maxi- mal power output (Wmax) for the group was $164 \pm 5 \mathrm{~W}$.

\section{HRV changes during the experiment}

No changes in the assessed time and frequency domain parameters were observed during the L-phase (using ANOVA contrasts) and therefore we chose the HRV parameters of the last segment (L5) as representative of the L-phase.

\section{Time domain parameters (Table 1, Figure 2)}

All time domain parameters changed significantly during the experiment $(\mathrm{P}<0.0005$, ANOVA). Compared to the L-phase, the mean RR interval decreased during the Sphase. After exercise mean RR interval gradually increased but throughout the $30 \mathrm{~min}$ of the R-phase analyzed it did not return to the pre-exercise supine (L-phase) value. A similar course of changes in SDRR, RMSSD and pNN50 was observed, with the most marked drop during the $\mathrm{S}$ - and R-phase found in pNN50.

\section{Frequency domain parameters (Table 1, Figure 3)}

All assessed frequency domain parameters changed significantly during the experiment $(\mathrm{P}<0.0005$, ANOVA). Compared to the L-phase, HF power decreased during the S-phase. However, neither a significant increase nor a decrease in LF power was observed when the subject changed his position from supine to standing. After exercise, both spectral powers (HF and LF) gradually increased but during the $30 \mathrm{~min}$ of the Rphase analyzed they did not attain their preexercise values.

\section{Heart rate complexity (Table 1, Figure 4)}

Sample entropy was significantly lower during standing in comparison with the L- 
Table 1 . Comparisons of heart rate variability and heart rate complexity indices using repeated measures ANOVA with contrasts.

\begin{tabular}{|c|c|c|c|c|c|c|c|c|c|c|}
\hline \multicolumn{11}{|l|}{ A } \\
\hline & Segment & \multicolumn{2}{|c|}{ L vs S } & \multicolumn{2}{|c|}{ L vs R1 } & L vs R2 & \multicolumn{2}{|c|}{ L vs R3 } & L vs R4 & L vs R5 \\
\hline lean RR & $<0.0005^{*}$ & \multicolumn{2}{|c|}{$<0.0005^{*}$} & \multicolumn{2}{|c|}{$<0.0005^{*}$} & $<0.0005^{*}$ & \multicolumn{2}{|c|}{$<0.0005^{*}$} & $<0.0005^{*}$ & $<0.0005^{*}$ \\
\hline SDRR & $<0.0005^{*}$ & \multicolumn{2}{|c|}{$0.015^{*}$} & \multicolumn{2}{|c|}{$<0.0005^{*}$} & $<0.0005^{*}$ & \multicolumn{2}{|c|}{$0.002^{*}$} & $0.005^{*}$ & $0.001^{*}$ \\
\hline RMSSD & $<0.0005^{*}$ & \multicolumn{2}{|c|}{$<0.0005^{*}$} & \multicolumn{2}{|c|}{$<0.0005^{*}$} & $<0.0005^{*}$ & \multicolumn{2}{|c|}{$0.001 *$} & $<0.0005^{*}$ & $<0.0005^{*}$ \\
\hline NN50 & $<0.0005^{*}$ & \multicolumn{2}{|c|}{$<0.0005^{*}$} & \multicolumn{2}{|c|}{$<0.0005^{*}$} & $<0.0005^{*}$ & \multicolumn{2}{|c|}{$<0.0005^{*}$} & $<0.0005^{*}$ & $<0.0005^{*}$ \\
\hline $\mathrm{g} \mathrm{HF}$ & $<0.0005^{*}$ & \multicolumn{2}{|c|}{$<0.0005^{*}$} & \multicolumn{2}{|c|}{$<0.0005^{*}$} & $<0.0005^{*}$ & \multicolumn{2}{|c|}{$<0.0005^{*}$} & $<0.0005^{*}$ & $<0.0005^{*}$ \\
\hline & $<0.000$ & \multicolumn{2}{|c|}{0.119} & \multicolumn{2}{|c|}{$<0.0005^{*}$} & $<0.000$ & \multicolumn{2}{|c|}{$0.002 *$} & $0.012^{*}$ & \\
\hline SampEn & $<0.0005^{*}$ & \multicolumn{2}{|c|}{$<0.0005^{*}$} & \multicolumn{2}{|c|}{0.111} & 0.047* & \multicolumn{2}{|c|}{$0.030^{*}$} & $0.043^{*}$ & 0.198 \\
\hline \multicolumn{11}{|c|}{ 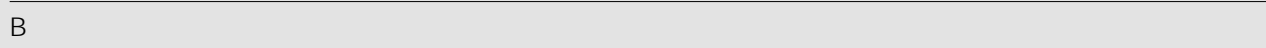 } \\
\hline & R1 vs R2 & R2 vs R3 & R3 vs & s R4 & R4 vs R5 & R1 vs R3 & $\mathrm{R} 2$ vs R4 & R3 vs R5 & R1 vs R4 & R2 vs R5 \\
\hline ean & $<0.0005^{*}$ & $0.001 *$ & & & $0.012 *$ & $<0.0005^{*}$ & $0.001 *$ & $0.005 *$ & $<0.0005^{*}$ & $<0.0005^{*}$ \\
\hline & $0.001 *$ & 0.074 & & & 0.521 & & $0.008^{*}$ & 0.324 & & $0.006^{*}$ \\
\hline SD & 0.210 & $0.017 *$ & 0.4 & & 0.695 & $0.008 *$ & $0.017 *$ & $0.047 *$ & $0.006 *$ & $0.002 *$ \\
\hline pNN50 & $0.021 *$ & 0.099 & 0.02 & 22* & 0.469 & $0.022 *$ & $0.008^{*}$ & $0.003^{*}$ & $0.004 *$ & $0.001^{*}$ \\
\hline $\log \mathrm{HF}$ & $0.005^{*}$ & $0.020^{*}$ & 0.12 & & 0.228 & 0.004* & $<0.0005^{*}$ & $0.017^{*}$ & $<0.0005^{*}$ & $<0.0005^{*}$ \\
\hline $\log L F$ & $0.001 *$ & 0.083 & 0.1 & & 0.802 & $0.001 *$ & $0.005^{*}$ & 0.156 & $0.001 *$ & $0.004 *$ \\
\hline SampEn & 0.989 & 0.583 & 0.8 & & 0.051 & 0.676 & 0.498 & 0.300 & 0.584 & 0.444 \\
\hline
\end{tabular}

The values in the table are probabilities associated with each comparison. A, The main effects of segment are given in the 1st column. The comparisons of the recovery vs the supine rest phase are presented thereafter. B, Comparisons during the recovery phase. L-, S-, R1-, R2-, R3-, R4-, R5-segments of analyzed record (for a more detailed description, see Material and Methods). Mean RR, mean duration of RR intervals; SDRR, standard deviation of RR intervals; RMSSD, square root of the mean squared difference of successive RR intervals; pNN50, proportion of interval differences of successive RR intervals greater than $50 \mathrm{~ms}$; log HF and $\log \mathrm{LF}, \log$ high and low frequency spectral powers, respectively; SampEn, sample entropy. ${ }^{*} \mathrm{P}<0.05$.
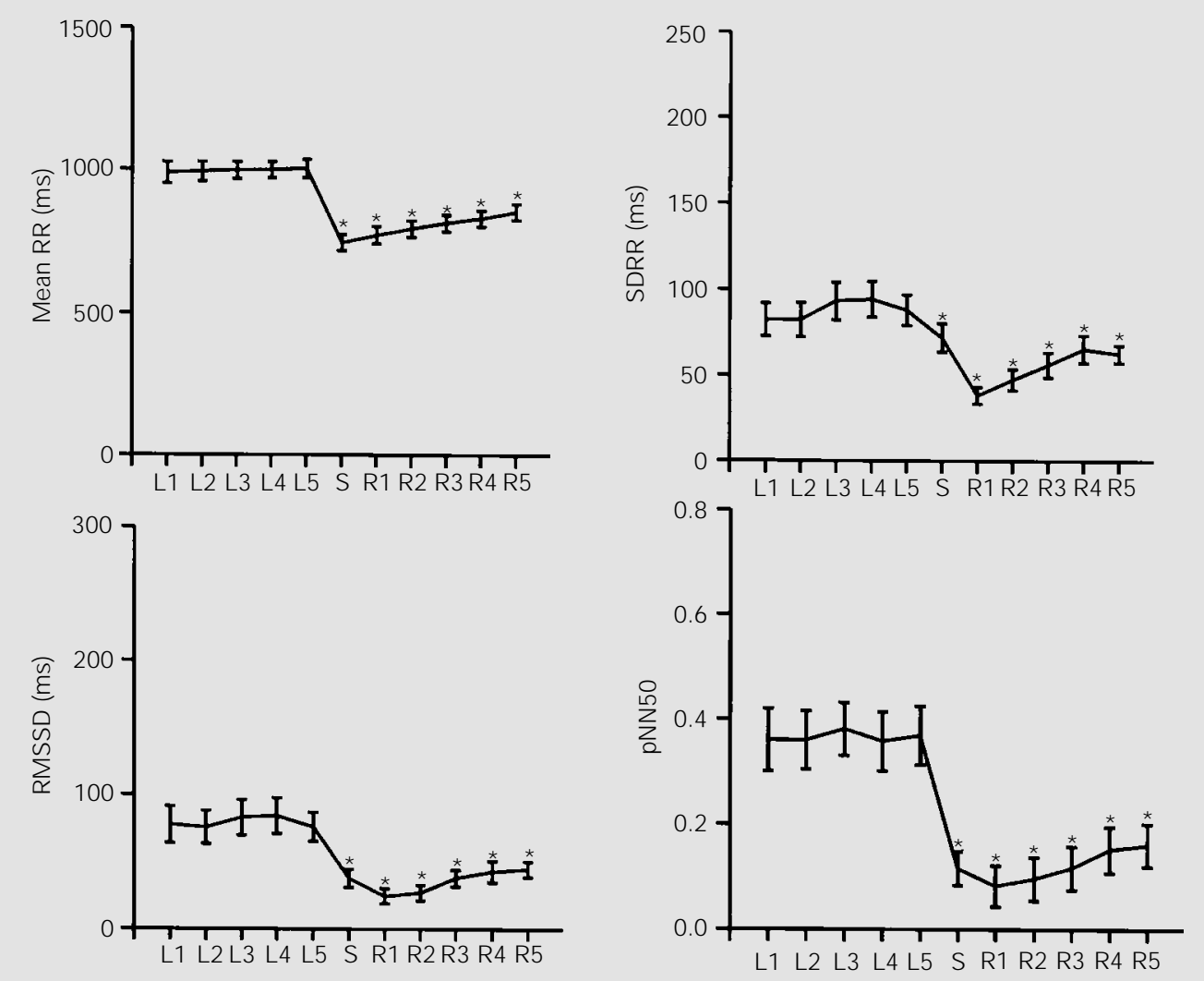

Figure 2. Changes of time domain heart rate variability indices during the experiment. Data are reported as means and the error bars represent the SEM. $* \mathrm{P}<0.05$ compared to L-phase (repeated measures ANOVA). For abbreviations see legend to Table 1. 
Figure 3. Changes of frequency domain heart rate variability indices during the experiment. Data are reported as means and the error bars represent the SEM. $* \mathrm{P}<0.05$ compared to L-phase (repeated measures ANOVA). LF, HF, low and high frequency spectral powers, respectively.

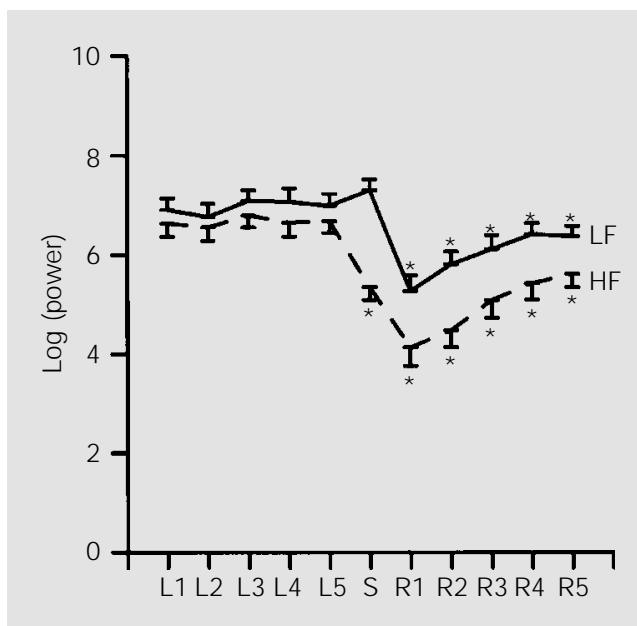

Figure 4. Changes of sample entropy (SampEn) during the experiment. Data are reported as means and the error bars represent the SEM. $* \mathrm{P}<0.05 \mathrm{com}$ pared to L-phase (repeated measures ANOVA)

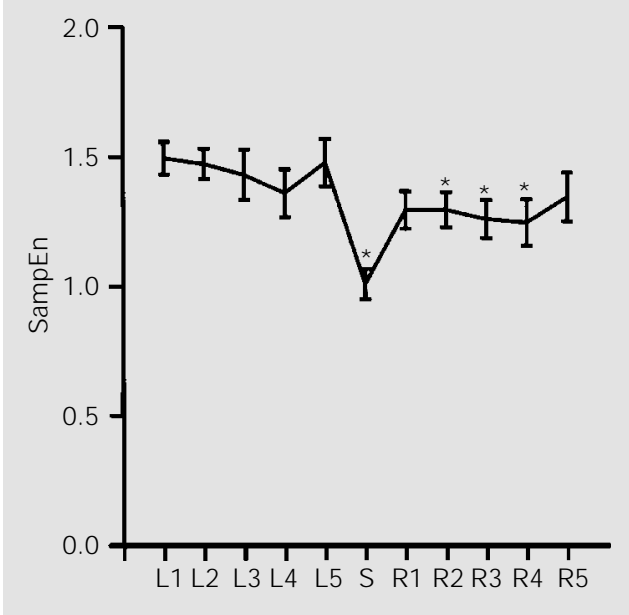

phase. During recovery, sample entropy was greater than during the S-phase and slightly (and significantly) lower than in the supine position before exercise. Only during the last segment analyzed, R5 (approximately 25-30 min after cessation of exercise), did sample entropy attain values not significantly different from the L-phase.

\section{Heart rate recovery and $\mathrm{HRV}$}

During the 1st minute of recovery, heart rate decreased by $38 \pm 9 \%$ of peak heart rate during exercise. No significant correlations (Table 2) between \%D1 and time, frequency domain HRV parameters or sample entropy from the L-phase and S-phase were found. However, significant positive correlations were observed between \%D1 and all assessed time and frequency domain parameters obtained from the R1 and R2 segments. In addition, a significant positive correlation between \%D1 and sample entropy computed from the R1 segment was found. Among the parameters assessed during the recovery period more than $15 \mathrm{~min}$ after the cessation of exercise (R3-R5 segments), only pNN50 showed a significant positive correlation with $\% \mathrm{D} 1$.

\section{Discussion}

The major findings of our study were: i) time and frequency domain HRV indices continuously increased during the recovery phase after exercise and remained reduced (compared to supine rest) for at least $30 \mathrm{~min}$; ii) heart rate complexity was markedly reduced in the standing position compared to supine rest and a slight reduction observed during the recovery phase returned to supine rest level after 30 min of supine recovery; iii) percent decrease of heart rate during the first minute of recovery was not correlated with HRV parameters assessed during the supine rest and standing phase, but were positively correlated with all HRV indices from the onset of recovery. 


\section{Autonomic nervous system, HRV and exercise}

During exercise, cardiovascular parameters change to supply oxygen to working muscles and to preserve perfusion of vital organs. The vascular resistance and heart rate are controlled differently during physical activity $(12,13)$. At the onset of exercise heart rate (and cardiac output) elevation is mediated mostly by central command signals via vagal withdrawal. As work intensity increases and heart rate approaches 100 beats/ $\mathrm{min}$, sympathetic activity begins to rise, further increasing heart rate and plasma norepinephrine concentration and vasoconstricting vessels in visceral organs $(2,13-15)$.

With cessation of exercise, loss of central command, baroreflex activation and other mechanisms contribute to a rise in parasympathetic activity, causing a decrease in heart rate despite maintained sympathetic activation (12). Later, sympathetic withdrawal after exercise was also observed (16).

Rhythmic fluctuations in efferent sympathetic and vagal activities directed at the sinus node manifest as HRV. Analysis of these oscillations may permit inferences on the state and function of various cardiovascular control components (9). It was frequently observed that overall HRV (represented by SDRR), LF and HF spectral powers and mean RR interval (reciprocal value of heart rate) are considerably reduced during exercise, a fact that makes spectral analysis hard to carry out for exercise. During recovery, HRV is gradually regained $(8,14$, 17). In agreement with previous results, we observed a parallel increase of all HRV indices during $30 \mathrm{~min}$ of supine recovery after the step test. HRV indices increased more rapidly during the first half of recovery and more slowly later. However, even after 30 min all the HRV parameters remained reduced compared to rest values in agreement with Takahashi et al. (18), who also reported reduced HF power after $10 \mathrm{~min}$ of post- exercise supine recovery.

Most fluctuations of the RR interval in humans are driven by fluctuations of vagalcardiac nerve traffic (19). In our study, all time domain HRV indices (SDRR - representing overall HRV, RMSSD and pNN50 quantifying beat-to-beat variability) changed in a similar way, i.e., they decreased upon standing and gradually increased during recovery after exercise. These HRV parameters represent changes in vagal activity during the experiment. From this point of view, changing position from supine to standing is characterized by a reduction of cardiac parasympathetic activity and this activity is increasingly regained during post-exercise recovery.

During exercise, the HF component of HRV was found to be a valid index of parasympathetic cardiac nerve activity because it decreased in response to increases in exercise intensity and was attenuated by cholinergic receptor inhibition (15). In agreement with Grasso et al. (20), our results showed a marked reduction of HF upon standing and a gradual increase during recovery, indicating parasympathetic reactivation after exercise.

More controversial is the interpretation of the LF component, which is considered by some to be a marker of sympathetic modulation and by others as a parameter that includes sympathetic, vagal and baroreflex influences $(9,20,21)$. In a meta-analysis of HRV studies, Eckberg (21) showed that vagal contributions to LF RR-interval fluctuations are great, and there is no convincing evidence that baseline LF RR-interval spectral power is related quantitatively to sympatheticcardiac nerve traffic. We observed no significant change in HRV LF upon standing. In addition, we found a gradual increase of LF of HRV during post-exercise recovery parallel to HF and time domain indices. Considering these data as a whole, we suggest that during recovery LF of HRV is predominantly influenced by changes of parasympathetic activity directly (through alterations 
of vagal-cardiac activity causing fluctuations in LF band) and/or indirectly (through changes of baroreflex sensitivity).

In principle, biological systems are nonlinear. Nonlinear dynamics analysis can be used as a powerful tool for the description of biosignal characteristics (22). Nonlinear parameters are able to reveal small differences in the behavior of systems. One of the recently introduced parameters, which is able to quantify regularity, predictability and complexity of analyzed time series (and systems) is approximate entropy, introduced by Steven Pincus in 1991 (11,23). Approximate entropy can be used as an index of control system complexity - lower approximate entropy values indicate higher autonomy of system components underlying the dynamics of the assessed parameter. On the other hand, approximate entropy increases with more complex interconnections within a system $(11,24)$. Richman and Moorman (10) improved its mathematical properties and this new measure of time series complexity was named sample entropy.

We observed a marked reduction of sample entropy with the change of position from supine to standing, which is in agreement with observations by Yeragani et al (25). This fact indicates the simplification of heart rate control in standing with predominance of LF rhythm after reduction of vagal influences on the heart following parasympathetic withdrawal and sympathetic activation (25). During post-exercise recovery sample entropy was slightly decreased compared to supine rest before exercise and regained supine rest values after $30 \mathrm{~min}$. Despite a markedly reduced HRV, heart rate dynamics after exercise was more complex than in the standing position. Based on changes of sample entropy, we assume that both divisions of the autonomic nervous system significantly influence heart rate during recovery after exercise. After 30 min, vagus activity is increased to the extent necessary for the return of system complexity to the supine rest value.

\section{Heart rate recovery after exercise and its relation to HRV}

The post-exercise exponential decline of heart rate is an intrinsic property of the intact circulation independent of autonomic control (4). Heart rate rapidly decreases during the first 1-2 min after the cessation of exercise, and gradually thereafter. During recovery from moderate and heavy exercise heart rate remains elevated above the pre-exercise level for a relatively long period of time (up to $60 \mathrm{~min})(2,18,26-28)$. Because of the presumed parasympathetic origin of both HRV and the rate of heart rate decrease after exercise we hypothesized that the HRV indices before and after exercise could be associated with the rate of heart rate recovery. Correlation analysis revealed that our hypothesis was not completely true: HRV during supine rest and standing was not related to the rate of post-exercise heart rate recovery; however, the significant positive correlations between \%D1 and all assessed time and frequency domain parameters obtained from the 5 th and 10th minute of recovery were observed. Therefore, the increase of parasympathetic activity causing heart rate deceleration after exercise is to a large extent independent of basal parasympathetic tone. From a clinical point of view, quantification of HRV during various maneuvers can provide additional information about cardiovascular system adaptability and flexibility with potential prognostic clinical application.

\section{Study limitations}

It is well known that the magnitude of neural and hemodynamic responses to exercise is related to exercise intensity (16). In our study we assessed cardiovascular parameters after exercise at $70 \%$ of individual maximal power output level. Thus, it is possible that different exercise intensities have also distinct effects on cardiovascular 
changes during and after exercise.

Since adaptation to exercise acquired by physical training can significantly influence the cardiovascular response to exercise (29), we performed this study on healthy untrained subjects.

HRV indices (and particularly HF spectral power) are to a large extent influenced by breathing pattern and it is usually recommended to control the frequency of breathing and tidal volume in HRV studies (30). We did not attempt to control breathing pattern in order to avoid subject's discomfort and metabolic and blood gas changes due to unwanted hypo- or hyperventilation. It was shown that minute ventilation, tidal volume and respiratory frequency gradually decrease during post-exercise recovery (27). The tidal volume drop could reduce the HF increase during recovery; on the other hand, the HF increase could be to some extent caused by the post-exercise respiratory frequency decrease. Therefore, the HF power changes should be interpreted with caution as alterations of vagal-cardiac activity.

In conclusion, we found that after exercise time and frequency domain HRV indices continuously increased during the recovery phase. The rate of heart rate decrease during recovery was not correlated with the HRV parameters obtained from supine rest and standing, but was positively correlated with all HRV indices obtained from the onset of recovery ( 5 and 10 min after the cessation of exercise). In addition, heart rate complexity was markedly reduced in the standing position and a slight reduction of sample entropy during the recovery phase returned to pre-exercise levels after $30 \mathrm{~min}$ of supine recovery.

\section{References}

1. Shephard R (1987). Exercise Physiology. B.C. Decker Inc., Philadelphia, PA, USA.

2. Carter III R, Watenpaugh DE, Wasmund WL, Wasmund SL \& Smith ML (1999). Muscle pump and central command during recovery from exercise in humans. J oumal of Applied Physiology, 87: 14631469.

3. Nishime EO, Cole CR, Blackstone EH, Pashkow FJ \& Lauer MS (2000). Heart rate recovery and treadmill exercise score as predictors of mortality in patients referred for exercise ECG. J ournal of the American Medical Association, 284: 13921398.

4. Savin WM, Davidson DM \& Haskell WL (1982). Autonomic contribution to heart rate recovery from exercise in humans. J ournal of Applied Physiology, 53: 15721575.

5. Chorbajian T (1971). Nomographic approach for the estimation of heart rate recovery time after exercise. J ournal of Applied Physiology, 31: 962-964.

6. Ashley EA, Myers J \& Froelicher V (2000). Exercise testing in medicine. Lancet, 356: 1592-1597.

7. Cole CR, Blackstone EH, Pashkow FJ, Snader CE \& Lauer MS (1999). Heart-rate recovery immediately after exercise as a predictor of mortality. New England J ournal of Medicine, 341: 1351-1357.

8. Salinger J, Opavský J, Stejskal P, Vychodil R, Olšák S \& J anura M (1998). The evaluation of heart rate variability in physical exercise by using telemetric Variapulse TF 3 system. Gymnica, 28: 13-23.

9. Task Force of the European Society of Cardiology and the North American Society of Pacing and Electrophysiology (1996). Heart rate variability. Standards of measurement, physiological interpretation, and clinical use. Circulation, 93: 1043-1065.

10. Richman J S \& M oorman J R (2000). Physiological time-series analysis using approximate entropy and sample entropy. American J ournal of Physiology, 278: H2039-H2049.

11. Pincus SM \& Goldberger AL (1994). Physiological time-series analysis: what does regularity quantify? American J ournal of Physiology, 266: H1643-H1656.

12. O'Leary D (1993). Autonomic mechanisms of muscle metaboreflex control of heart rate. J oumal of Applied Physiology, 74: 1748-1754.

13. Rowell LB \& O'Leary DS (1990). Reflex control of the circulation during exercise: chemoreflexes and mechanoreflexes.
J oumal of Applied Physiology, 69: 407418.

14. Kluess HA, Wood RH \& Welsch MA (2000). Vagal modulations of the heart and central hemodynamics during handgrip exercise. American J ournal of Physiology, 279: H1648-H1652.

15. Warren J H, J affe RS, Wraa CE \& Stebbins CL (1997). Effect of autonomic blockade on power spectrum of heart rate variability during exercise. American J oumal of Physiology, 273: R495-R502.

16. Forjaz CLM, Matsudaira Y, Rodrigues FB, Nunes N \& Negrão CE (1998). Post-exercise changes in blood pressure, heart rate and rate pressure product at different exercise intensities in normotensive humans. Brazilian J ournal of Medical and Biological Research, 31: 1247-1255.

17. Oida E, Moritani T \& Yamori Y (1997). Tone-entropy analysis on cardiac recovery after dynamic exercise. J ournal of Applied Physiology, 82: 1794-1801.

18. Takahashi T, Okada A, Saitoh T, Hayano J $\&$ Miyamoto $Y$ (2000). Difference in human cardiovascular response between upright and supine recovery from upright cycle exercise. European J oumal of Applied Physiology, 81: 233-239.

19. Eckberg DL (2000). Physiological basis for 
human autonomic rhythms. Annals of Medicine, 32: 341-349.

20. Grasso R, Schena F, Gulli G \& Cevese A (1997). Does low-frequency variability of heart period reflect a specific parasympathetic mechanism? J ournal of the Autonomic Nervous System, 63: 30-38.

21. Eckberg DL (1997). Sympathovagal balance. A critical appraisal. Circulation, 96: 3224-3232.

22. Fusheng $Y$, Bo $H \& \&$ Qingyu $T$ (2001). Approximate entropy and its application in biosignal analysis. In: Akay M (Editor), Nonlinear Biomedical Signal Processing. Vol. II: Dynamical Analysis and Modeling. IEEE Press, New York, NY, USA.

23. Pincus SM (1991). Approximate entropy as a measure of system complexity. Pro- ceedings of the National Academy of Sciences, USA, 88: 2083-2088.

24. Pincus SM (2000). Approximate entropy in cardiology. Herzschrittmachertherapie und Elektrophysiologie, 11: 139-150.

25. Yeragani VK, Srinivasan K, Vempati S, Pohl R \& Balon R (1993). Fractal dimension of heart rate time series: an effective measure of autonomic function. J oumal of Applied Physiology, 75: 2429-2438.

26. Brown SP, Li H, Chitwood LF, Anderson ER \& Boatwright D (1993). Blood pressure, hemodynamic, and thermal responses after cycling exercise. J ournal of Applied Physiology, 75: 240-245.

27. Miyamoto $Y$, Hiura T, Tamura T, Nakamura T, Higuchi J \& Mikami T (1982). Dynamics of cardiac, respiratory, and metabolic func- tion in men in response to step work load. J oumal of Applied Physiology, 52: 11981208.

28. Takahashi T, Okada A, Hayano J , Tamura T \& Miyamoto Y (2000). Influence of duration of cool-down exercise on recovery of heart rate in humans. Therapeutic Research, 21: 48-53.

29. Hagberg J M, Hickson RC, Ehsani AA \& Holloszy J O (1980). Faster adjustments to and recovery from submaximal exercise in the trained state. J ournal of Applied Physiology, 48: 218-224.

30. Brown TE, Beightol LA, Koh J \& Eckberg DL (1993). Important influence of respiration on human R-R interval power spectra is largely ignored. J oumal of Applied Physiology, 75: 2310-2317. 